\title{
Chromaticity of the lattice and beam stability in energy-recovery linacs
}

\author{
V.N. Litvinenko
}

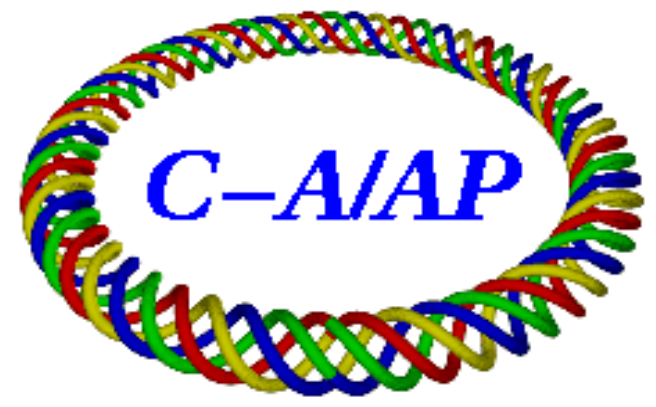

\section{Collider-Accelerator Department Brookhaven National Laboratory Upton, NY 11973}

Notice: This document has been authorized by employees of Brookhaven Science Associates, LLC under Contract No. DE-AC02-98CH10886 with the U.S. Department of En ergy. The United States Government retains a nonexclusive, paid-up, irrevocable, world-wide license to publish or reproduce the published form of this document, or allow others to do so, for United States Government purposes. 


\title{
Chromaticity of the lattice and beam stability in energy-recovery linacs
}

\author{
Vladimir N. Litvinenko* \\ Brookhaven National Laboratory, Upton, NY, USA \\ Department of Physics and Astronomy, Stony Brook University, Stony Brook, NY, USA
}

\begin{abstract}
Energy recovery linacs (ERLs) are an emerging generation of accelerators promising to revolutionize the fields of high-energy physics and photon sciences. These accelerators combine the advantages of linear accelerators with that of storage rings, and hold the promise of delivering electron beams of unprecedented power and quality. Use of superconducting radio-frequency (SRF) cavities converts ERLs into nearly perfect "perpetuum mobile" accelerators, wherein the beam is accelerated to a desirable energy, used, and then gives the energy back to the RF field. One potential weakness of these devices is transverse beam break-up instability that could severely limit the available beam current. In this paper, I present a method of suppressing these dangerous effects using a natural phenomenon in the accelerators, viz., the chromaticity of the transverse motion.
\end{abstract}

\section{Introduction}

Energy recovery linacs (ERLs, see Fig.1) belong to a family of recirculating linacs (RL) that accelerate a beam of charged particles multiple times in the same linear accelerator, and accumulate the beam's energy on each pass. The difference between an $\mathrm{RL}$ and an ERL is that the latter does not dispose of the accelerated beam, but instead recovers its energy by decelerating the beam down to the injection energy (Figure 1).

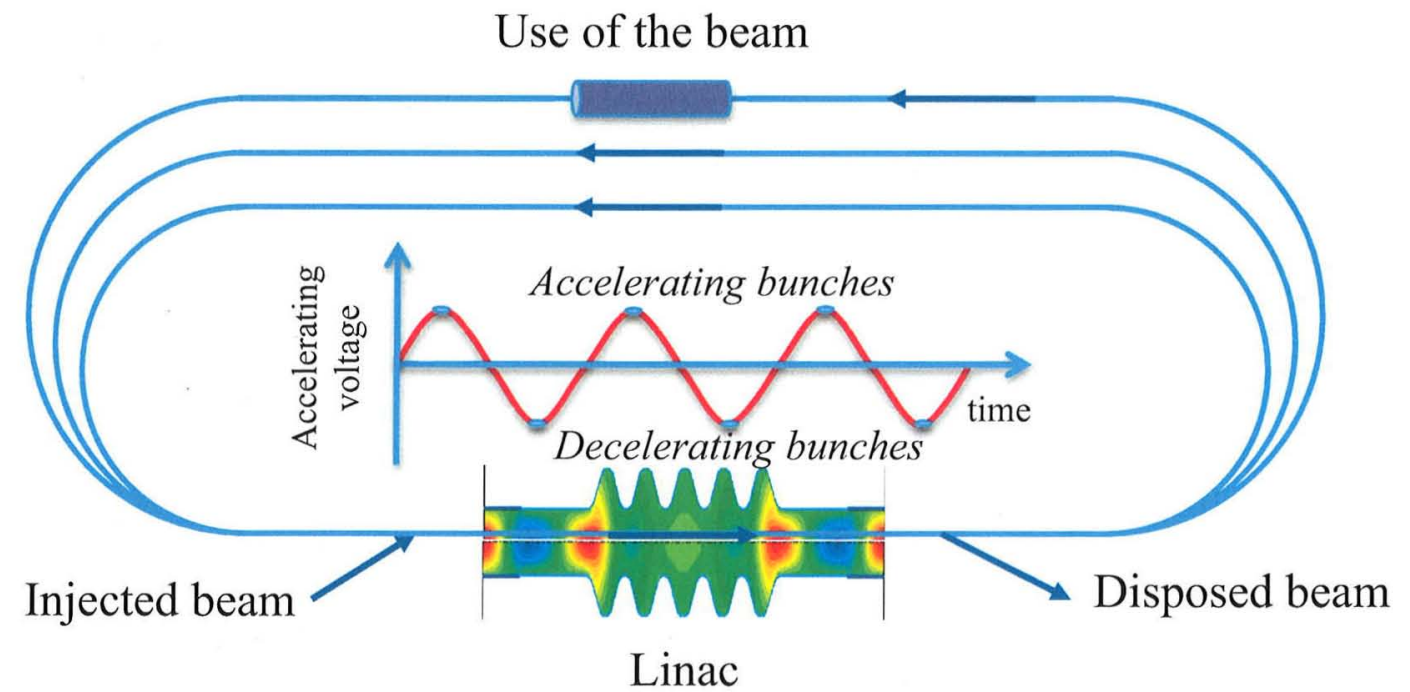

Figure 1. A simplified sketch of a three-pass ERL comprised of the linear accelerator and three returning loops.

* Corresponding author vl@bnl.gov 
In ERL low-energy particles are injected and accelerated on the crest (or near the crest) in the SRF linac to the top energy. After their usage at top energy, the beam's RF phase is shifted by 180 degrees, and the beam is decelerated in the same linac. The later provides for the recovery of the beam energy into that of the EM field in the linac. Finally, the beam is ejected and disposed at a very low energy compared with the top energy.

In 1965, Tigner suggested the idea of an energy recovery linac [1]. It took some thirty-five years of development of superconducting radiofrequency (SRF) ERLs to reach beam currents $\sim 10 \mathrm{~mA}$, and energy of few hundreds $\mathrm{MeV}$. While this sufficed for the first spectacular demonstration of generating very high powerful coherent-radiation in $2000[2,3]$ at the Thomas Jefferson Accelerator Facility (JLab), there is a well-established need for ERLs with GeV-scale energy, and ampere-scale currents in fundamental- and applied-sciences. Such ERLs would find unique applications for next-generation light sources [4,5] and similarly for high-energy physics colliders [6-8].

One main challenge toward achieving these goals is the transverse beam break-up instability (TBBU) that is especially severe for SRF recirculating linacs. The extent of this problem was recognized in early experiments with recirculating SRF accelerators at Stanford [9] and Illinois [10], where this instability was occurring at a few microamperes of the average beam current. Dipole high-order modes (HOMs) of the SRF cavities were identified as the culprits driving this instability [11-12], and several remedies were developed for raising this threshold [13].

Detailed theoretical approaches and simulation programs were developed in the late 80s [14-16]. The renewed interest in this problem, the refinements of the TBBU theory, the simulation programs [17-19] and their experimental verification [20] all are driven by the need for high current ERLs, and also by the rewards of resolving this complex problem. Strong damping of HOMs in SRF linacs while maintaining high accelerating gradients remains one of the major unsolved issues.

In this paper, I suggest a novel method of addressing TBBU instability by using a naturally occurring phenomenon, i.e., the chromaticity ${ }^{1}$ of the ERL's return loops.

Before discussing the working of the proposed suppression mechanism, I reiterate the fundamentals of TBBU instability, sketched in Figure 2.

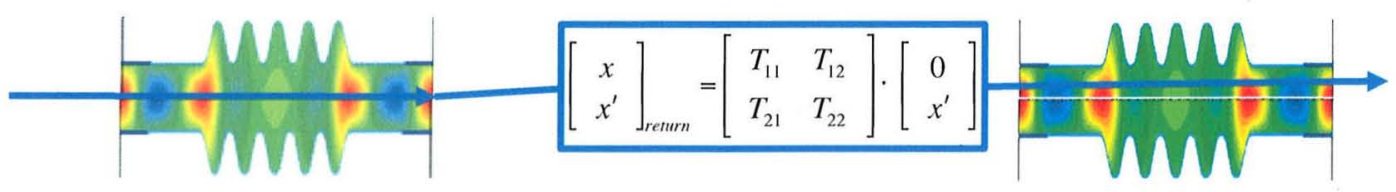

Figure 2. A sketch of the driving mechanism of TBBU instability in ERLs.

\footnotetext{
${ }^{1}$ Chromaticity is a natural phenomenon caused by the energy dependence of the focusing strength of the magnetic elements in the ERL's loops.
} 
Let's consider the SRF cavity with residual oscillations of a dipole transverse (TM) HOM that gives a transverse kick to the passing bunch (indicated by $x^{\prime}$ in Fig.2). When the bunch returns to the entrance of the same cavity, it acquires a transverse displacement that is proportional to the $T_{12}$ element of the transport matrix (map) of the returning loop:

$$
x_{r}=T_{12} \cdot x^{\prime}
$$

which excites the field of the HOM${ }^{2}$. The strength the HOM's longitudinal electric field is proportional to its distance from the axis. For some of the many HOMs, the phasing can be such that the bunch sees a decelerating electric field and looses part of its energy; this energy is transferred into that of the HOM field. The next bunch in the beam would receive a stronger kick, will come back with a larger transverse-displacement, and would further excite the cavity. Hence, without the decay of the HOM field, such a system is prone to instability. The SRF cavities provide an environment wherein the $Q$-factor can reach values $\sim 10^{8}-10^{9}$, i.e., the decay times of the HOMs can be measured in seconds; thus, reducing the HOMs' $Q$-factors to the required values, $Q \sim 10^{3}$, is a very challenging problem [21].

In the general ERL case, one can write a complete set of dispersion relations in a matrix form [14-19], but an analytical solution of the TBBU's instability threshold can be derived only for a single HOM mode, and a single-pass ERL, and as detailed in [12]:

$$
I_{h h}=\frac{2 c^{2}}{e R_{g} \cdot Q \cdot \omega} \cdot \frac{1}{T_{12} \sin \omega t_{r}}
$$

where $c$ is the speed of the light, $e$ is the elementary charge, $R_{g} \cdot Q$ is the HOM's impedance (measured in $\Omega)^{3}, \omega$ is its frequency, and $t_{r}$ is the beam's travel time through the returning loop. Thus, for $T_{12} \sin \omega t_{t}>0$, the $I_{t h}>0$ and TBBU instability occurs for beam current above the threshold, i.e. $I>I_{t h}$. What is important that, for a given cavity with $2 c^{2} / e R_{g} \omega=$ const, the threshold current is inversely proportional to $Q \cdot\left|T_{12} \sin \omega t_{r}\right|$.

Since there are many HOM modes in the cavities covering a large range of the spectrum, there can be modes with $\left|\sin \omega t_{r}\right| \sim 1$; then, the only meaningful way of increasing the threshold is by reducing the values of $Q$ and the $\left|T_{12}\right|$.

As shown in [17], eq. (66), in an ERL with $N$ passes through its linac the TBBU threshold can be estimated by

\footnotetext{
${ }^{2}$ I focus here on the simple case of uncoupled motion in the loop; hence, the suppression mechanism does not require transverse coupling. As shown in [19], for a dipole HOM polarized at an arbitrary angle $\theta$ and arbitrary transverse coupling described by $4 \times 4$ transport matrix $T, T_{12}$ should be replaced in the TBBU dispersion relation by $T_{12} \cos ^{2} \theta+\left(T_{14}+T_{32}\right) \sin \theta \cos \theta+T_{34} \sin ^{2} \theta$. Since all consideration for $T_{12}$ are applicable to $T_{12}, T_{14}, T_{32}, T_{34}$, the suppression of the beam response by chromaticity will stay intact.

${ }^{3}$ The geometric impedance, $R_{g}$, is a function of the cavity's geometry and does not depend on the material (i.e., it does not depend on $\mathrm{Q}$ ). However, the RF literature frequently uses a confusing form $R_{g}=R / Q$.
} 


$$
I_{t h}=\frac{2 c^{2}}{e R_{g} \cdot Q \cdot \omega} \cdot \frac{1}{\sum_{J=1}^{2 N} \sum_{I=J+1}^{2 N} T^{l J} \sin \omega\left(t_{l}-t_{J}\right)}
$$

where $T^{\prime J} \equiv T_{12}\left(s_{J} \mid s_{I}\right)$ is the element of transport matrix between $J^{\text {th }}$ and $I^{t h}$ pass through the linac. Analyzing eq. (3) the authors of [17] come to a natural conjecture that the TBBU threshold scales as following:

$$
I_{t h} \propto\left(R_{g} \cdot Q \cdot N^{2}\left\langle\left|T^{l J}\right|\right\rangle\right)^{-1}
$$

One can argue that the linac HOM impedance scales with it length and therefore, for a fixed top energy of ERL, the geometrical impedance scales as $R_{g} \sim E_{\text {top }} / N$, and

$$
I_{t h} \propto\left(\tilde{R}_{g} \cdot Q \cdot N\left\langle\left|T^{I J}\right|\right\rangle\right)^{-1}
$$

where $\tilde{R}_{g}$ is HOM impedance for a unit length of the linac. On the other hand, typical values of $\beta$-functions, and therefore the typical values of $T^{I J}$, in the linac are proportional to its length (see footnote 7). This observation can bring back $N^{-2}$ dependence of the TBBU threshold.

In any case, this unfavorable scaling may have major implications on the cost of high energy ERL. Since SRF linac is usually more expensive than magnetic elements, the cost effective solutions [22-24] lead to a three to six pass ERLs. If such ERL designs suffer from severe current limitation, the extend of their use and their energy reach will be limited.

One way of solving these limitation is reducing $Q$ of all dangerous HOM by developing complicated HOM damping schemes and, in addition, limiting number of cells per linac module to avoid trapped high-Q HOMs [25-27]. These stringent requirements on HOM suppression frequently complicate the SRF linac designs, reduce the real-estate gradient and make them more expensive.

The other way of increasing the threshold current is reducing $\left\langle\left|T^{I I}\right|\right\rangle$. The latter is the topic of this paper, where I describe how the proposed suppression mechanism works.

\section{Suppression of the beam's response using the chromaticity of the ERL lattice}

Since the bunch is an ensemble of particles, its transverse response to the kick will be the average if the responses of the individual particles are

$$
\left\langle x_{r}\right\rangle=\left\langle T_{12}\right\rangle \cdot x^{\prime}
$$

where $\langle a\rangle$ signifies the average value of a parameter, $a$. I suggest using the chromaticity of the returning loops and energy spread in the beam to reduce $\left\langle T_{12}\right\rangle \rightarrow 0$.

In contrast to storage rings wherein the beam circulates for trillions of turns, in an ERL the beam passes through the system only once on the way up in energy, and once on 
its way down. In storage rings, chromaticity must be compensated for assuring both the beam's stability and a reasonable lifetime, while in ERLs this is not necessary, and, as discussed in this paper, may even be counterproductive. Hence, I consider a linear ERL lattice without sextupole fields ${ }^{4}$.

Let's consider transverse motion of an ultra-relativistic particle with momentum $p=p_{o}(1+\delta) ;|\delta| \ll<$ propagating through the linear transport system of the returning loop with a designed momentum, $p_{0}$, which is described by Hill's differential equation ${ }^{5}$ :

$$
x^{\prime \prime}=\frac{d^{2} x}{d s^{2}}=-\frac{K_{1}(s)}{1+\delta} \cdot x \cong-(1-\delta) K_{1}(s) \cdot x
$$

where $s$ is the longitudinal coordinate along the designed trajectory, and $K_{1}(s)$ is the rigidity of the transverse focusing for particle with the design energy [28]. The solution of this equation is well known in accelerator literature as the Courant-Snyder parameterization [29]:

$$
\begin{aligned}
& x=a \mathrm{w}(s) \cos (\psi(s)+\varphi) ; \psi^{\prime}=1 / \mathrm{w}^{2} ; \\
& x^{\prime}=a \mathrm{w}^{\prime}(s) \cos (\psi(s)+\varphi)-\frac{a}{\mathrm{w}(s)} \sin (\psi(s)+\varphi),
\end{aligned}
$$

where $a$ and $\varphi$ are constants of motion, and both the envelope function $w(s, \delta)$ and $\psi(s, \delta)$ depend on the particle's momentum ${ }^{6}$. Taking the kick at $s=0$ as the initial condition, $x_{0}=0 ; x_{0}^{\prime}=x^{\prime}$, yields

$$
\begin{gathered}
\varphi=-\pi / 2 ; \quad a=\mathrm{w}_{\mathrm{i}} x^{\prime} ; \mathrm{w}_{\mathrm{i}} \equiv \mathrm{w}(0) ; \\
x=x^{\prime} \cdot \mathrm{w}_{\mathrm{i}} \mathrm{w}(s) \sin \psi(s) .
\end{gathered}
$$

If all particles in the beam have the same energy, $E_{o}$, they will respond identically to the kick with $T_{12}(0 \mid s)=\mathrm{w}_{\mathrm{j}} \mathrm{w}(\mathrm{s}) \sin \psi(s) .^{7}$ For a particle with a small energy deviation, $|\delta| \ll<$, the envelope function and the phase advance can be extended as

$$
\mathrm{w}(s, \delta) \cong \mathrm{w}_{\mathrm{o}}(s)(1+\delta \cdot v(s)) ; \psi(s, \delta) \cong \psi_{\mathrm{o}}(s)+\delta \cdot \phi(s),
$$

${ }^{4}$ One can consider using nonlinear elements (sextupoles and octupoles) to introduce phase advance dependence on betatron action, i.e. a square of the amplitude of betatron oscillations. While possibly valid, such method would require introducing significant nonlinear field components on the scale of the transverse beam size and can negatively affect the beam quality.

${ }^{5}$ Throughout this paper I use the notations ' $\equiv \frac{d}{d s} ;{ }^{\prime \prime} \equiv \frac{d^{2}}{d s^{2}}$.

${ }^{6}$ I use the simple case of constant particle energy and momentum connected by a standard relativistic relation $E^{2}=p^{2} c^{2}+m^{2} c^{4}$, see [28] for a case of varying energy.

${ }^{7}$ The simple approach of choosing $\sin \psi(s)=0$ is possible only for a single cavity ERL. In practice, the length of the linacs, $L$, is rather large in any high-energy ERL linac ( $L \sim 100 \mathrm{~m}$ for a $1 \mathrm{GeV}$ SRF linac) and the average value of $\left|T_{12}\right|$ is comparable to the length of the linac, i.e., $\max \left(\left|T_{12}\right|\right)_{\text {linac }} \sim L$. 
where the subscript "o" indicates values for particles with the designed energy. The straightforward perturbation theory (see Appendix A and Appendix C) yields the following expression:

$$
\begin{aligned}
& \phi(s)=-\frac{1}{2} \int_{0}^{s} K(z) \mathrm{w}_{0}^{2}(z)\left(1+\cos 2\left(\psi_{0}(z)+\varphi_{0}\right)\right) d z \\
& v(s)=-\frac{1}{2} \int_{0}^{s} K(z) \mathrm{w}_{0}^{2}(z) \cdot \sin 2\left(\psi_{o}(z)+\varphi_{0}\right) d z
\end{aligned}
$$

One important (and well known) feature of this solution is that the phase deviation (i.e., the chromaticity of oscillations) is defined by a monotonic function with average rate of $-\left\langle K(z) \mathrm{w}^{2}{ }_{\mathrm{o}}(z)\right\rangle_{z} / 2$, while the envelope deviation is a fast oscillating function with double betatron frequency. Hence, in a large accelerator system, the chromaticity grows steadily, can reach significant values

$$
C(s)=\frac{\phi(s)}{2 \pi}>>1,
$$

and even for a modest deviation in relative energy, the phase variation can be large, while the relative variation in the envelope function remains small

$$
\delta \cdot v(s)<<1 .
$$

The later assures absence of the beam emittance growth in chromatic transport line.

Let us consider a bunch with Gaussian energy-distribution with a relative RMS energy spread $\sigma_{\delta}^{8}$ :

$$
f(\delta)=\frac{1}{\sqrt{2 \pi} \sigma_{\delta}} \exp \left(-\frac{\delta^{2}}{2 \sigma_{\delta}^{2}}\right)
$$

and then calculate value of the electron bunch displacement, i.e. $\langle x\rangle$ :

$$
\langle x(s)\rangle=x^{\prime} \cdot \mathrm{w}_{\mathrm{i} 0} \int_{-\infty}^{\infty} \mathrm{w}(s, \delta) \sin \psi(s, \delta) f(\delta) d \delta .
$$

Substituting (10) the integral (15) is easily evaluated:

$$
\left\langle T_{12}\right\rangle=\frac{\langle x(s)\rangle}{x^{\prime}}=\exp \left(-\frac{\left(\phi \sigma_{o}\right)^{2}}{2}\right) \cdot \mathrm{w}_{\mathrm{io}} \mathrm{w}_{\mathrm{o}}(s)\left(\sin \psi_{\mathrm{o}}(s)+v(s) \phi(s) \sigma_{\delta}^{2} \cos \psi_{\mathrm{o}}(s)\right) \text {. }
$$

with the suppression factor exponentially dependent on the chromaticity and the energy spread:

${ }^{8}$ Such a beam can be produced in an ERL injector from a cold beam generated from photoinjectors. For example, creating an electron bunch at the photocathode with Gaussian longitudinal profile, and then accelerating it off-crest will assure the desirable distribution. See detailed description of the method in Section III. 


$$
\left\langle T_{12}\right\rangle \propto \exp \left(-\frac{\left(\phi \sigma_{\delta}\right)^{2}}{2}\right) \cdot T_{12}(\max ) .
$$

Figures 4 and 5, below, demonstrate in a simplest form the effect of chromaticity on the response of the electron beam on a transverse momentum kick. More detailed examples are presented in section IV.

(a)

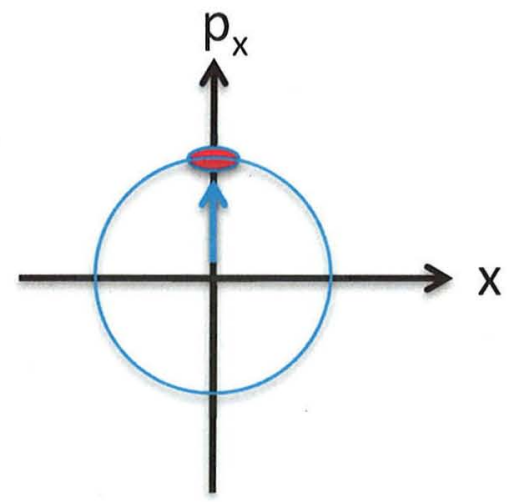

(b)

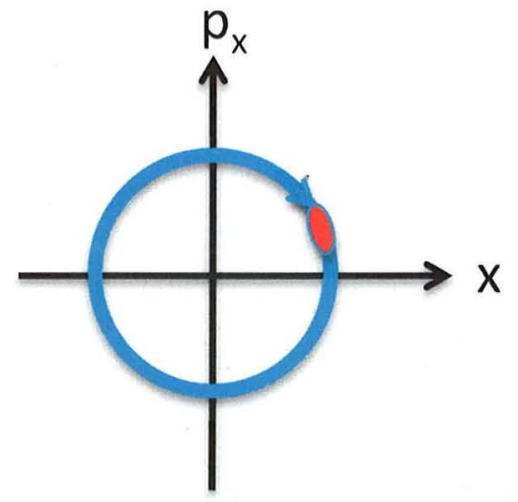

Fig. 4. In an arc with zero chromaticity, a transverse momentum kick received from the linac (a), will translate (after rotation in the phase space) into a displacement of the entire bunch at the exit of the transport channel (arc) (b).
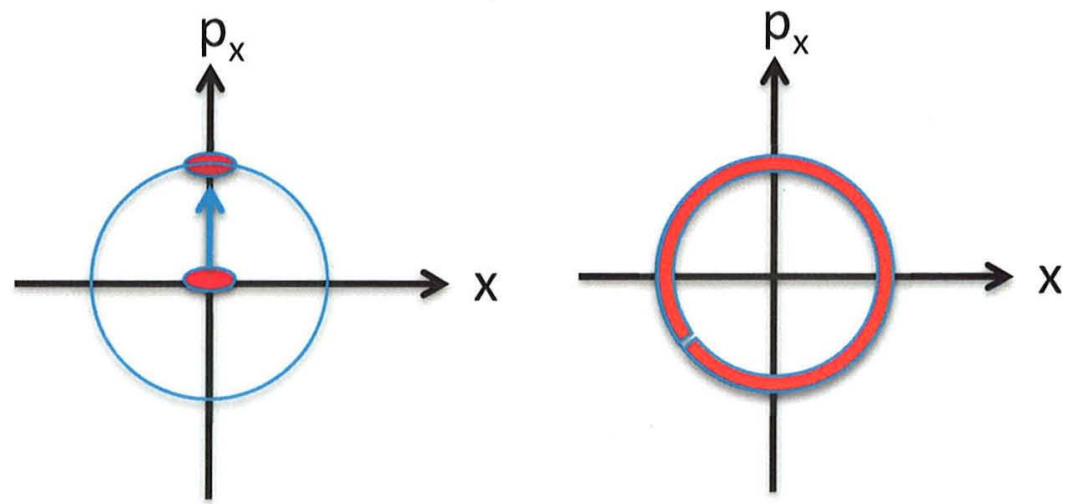

Fig. 5. In an arc with large chromaticity, a transverse momentum kick received from linac (a) will not displace the bunch overall because of the dependence of the phase-space rotation angle on the particles' energy spreads particle's displacement evenly around the circle (b).

Assuming a strong focusing lattice for return loops comprised of many periodic cells, similar to that designed for eRHIC electron-hadron collider [25], the loops' chromaticity can be $C(s) \sim-300$ and $\phi(s) \sim 2 \cdot 10^{3}$. Then, for a Gaussian beam with RMS energy spread of $0.2 \%$, the response $\left\langle T_{12}\right\rangle$ will be suppressed 3,000-fold, and according to 
formula (2) the threshold for TBBU instability will increase about this value or will be limited by other type of instability.

While the chromaticity can exponentially suppress the dipole moment excited in the beam by a kick, it does not caused the growth of the beam emittance - see Appendix A.

Naturally, the level of suppression of the beam's response (transfer function, $\left\langle T_{12}\right\rangle$ ) will depend on the energy-distribution function; Table 1 gives a few examples.

Table 1. Suppression of the beam's response on a transverse kick by the chromaticity, $\mathrm{C}$

In this table $X=\phi \sigma_{\delta} ; Y=v \sigma_{\delta}$.

\begin{tabular}{|c|c|c|}
\hline$f(\delta)$ & Suppression factor $\left\langle T_{12}\right\rangle / \mathrm{w}_{\mathrm{io}} \mathrm{w}_{\mathrm{o}}$ & Name \\
\hline$\frac{1}{\sqrt{2 \pi} \sigma_{\delta}} \exp \left(-\frac{\delta^{2}}{2 \sigma_{\delta}^{2}}\right)$ & $e^{-X^{2} / 2} \cdot(\sin \psi+X \cdot Y \cos \psi)$ & Gaussian \\
\hline$\frac{1}{\pi \sigma_{\delta}}\left(1+\frac{\delta^{2}}{\sigma_{\delta}^{2}}\right)^{-1}$ & $e^{-|X|} \cdot(\sin \psi+Y \cdot \operatorname{sign}(X) \cos \psi)$ & Lorentzian \\
\hline$\frac{2}{\pi \sigma_{\delta}}\left(1+\frac{\delta^{2}}{\sigma_{\delta}^{2}}\right)^{-2}$ & $e^{-|X|} \cdot((1+|X|) \sin \psi+X \cdot Y \cos \psi)$ & $\kappa-2$ \\
\hline$\frac{1}{2 \sigma_{\delta}}\left(\theta\left(\delta-\sigma_{\delta}\right)-\right.$ \\
$\left.\theta\left(\delta+\sigma_{\delta}\right)\right)$ & $\frac{\sin X}{X} \sin \psi+Y \frac{\sin X-X \cos X}{X^{2}} \cos \psi$ & Rectangular \\
\hline$\frac{1-\delta / \sigma_{\delta} \mid}{\left|\frac{\sigma_{\delta}}{\sigma_{\delta}}\right| \delta \mid \leq \sigma_{\delta}}$ & $2\left(\frac{\cos X-1}{X^{2}} \sin \psi+Y \cdot \frac{2(\cos X-1)+X \sin X}{X^{3}} \cos \psi\right)$ & Triangular \\
\hline
\end{tabular}

Smooth bell-shape energy-distribution functions (Gaussian, Lorentzian, $\kappa-2$ ) provide for a strong suppression of $\left\langle T_{12}\right\rangle$ whose value falls fast with the increase of $|X| \equiv 2 \pi|C| \sigma_{\delta}$, while sharp-edged distribution functions (such as a rectangular- or triangular-one) cause the oscillating behavior of $\left\langle T_{12}\right\rangle$ which declines slower with growth of $|X|$. In all cases highly chromatic lattice resulting in $|X|>>1$ provides for a effective method of suppressing the beam's response to transverse kicks.

\section{Practical issues of the de-phasing}

In some cases, there is enough chromaticity in an arc and energy spread in the electron beam to suppress the beam's response. If this this is not so, one can artificially create such a response suppressor using two RF cavities: One to create an energy spread at the entrance of the arc; the other to remove it at its end (Figure 6). Since most ERLs 
use isochronous arcs (i.e., arcs with $T_{56}=0$ ), neither the energy spread nor the beam's bunch length would be affected by such system. The only effect will be the introduction of additional energy spread in the arc, thereby suppressing the beam's response.

Such system has many advantages. One is that electron beam generated from photoinjectors frequently have Gaussian longitudinal distribution, and a linear chirp in the RF cavity will introduce Gaussian energy-spread in the beam. This means that for $\phi(s) \equiv 2 \pi C \sigma_{\delta} \gg>1$, the beam's response would be completely (exponentially) suppressed.

This scheme provides a practical way to suppress TBBU instability in almost all ERLs. ${ }^{9}$

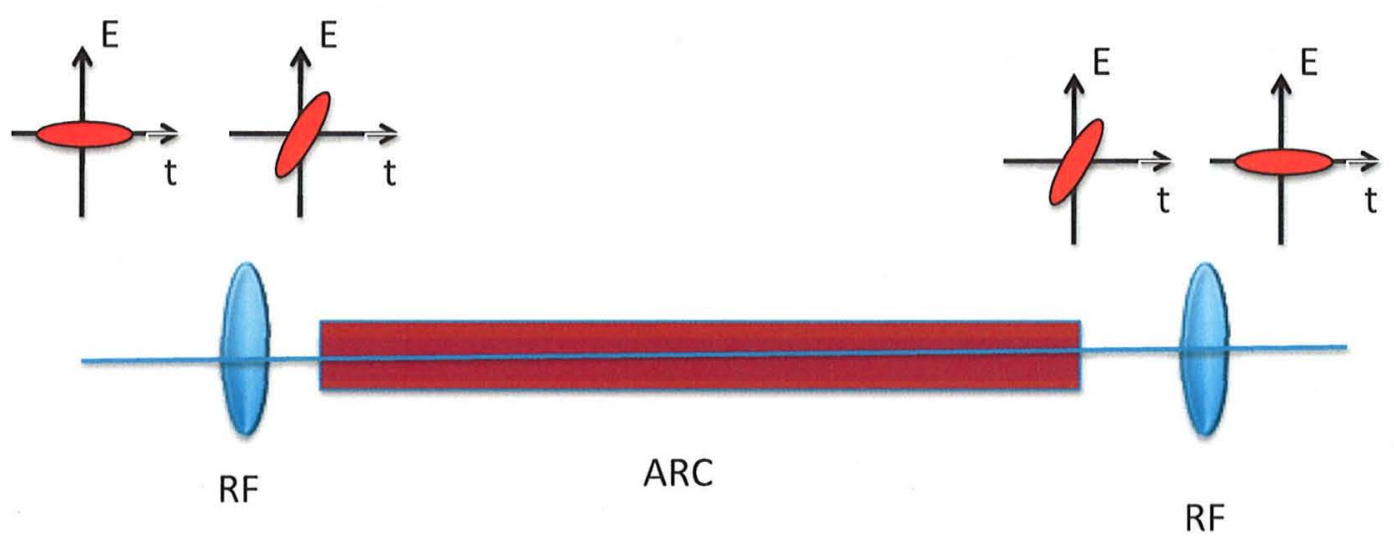

Figure 6. A sketch of a dedicated TBBU suppressor: One RF cavity gives an energy chirp to the electron beam at the entrance to the arc, and the other cavity removes the chirp.

\section{Examples}

Let's consider two specific examples of multi-pass ERLs proposed for electronhadron colliders eRHIC and LHeC. Their low-emittance achromatic arc lattices are described in two recent papers [30,31].

eRHIC's $30 \mathrm{GeV}$ ERL, shown in Fig. 7, comprises $0.6 \mathrm{GeV}$ injector and two 2.45 $\mathrm{GeV}$ super-conducting RF linacs. All eRHIC linacs work in energy recovery mode: the injector is a single path ERL, while the main linacs are part of 6-pass ERL. The electron beam from a $10 \mathrm{MeV}$ pre-injector is accelerated to $0.6 \mathrm{GeV}$ in the injector-ERL and than accelerated further to $30 \mathrm{GeV}$ passing six times through the main SRF linacs. After

\footnotetext{
${ }^{9}$ In the case of the eRHIC ERL with 6 passes [8], the energy of the electrons would affect its spin precession, and preserving the polarization of the electron beam would require alternating the sign of the chirp on consecutive passes. Since one arc in eRHIC is twice longer that the other and therefore has twice larger chromaticity, using the same amplitude of the chirp, would keep most the "memory loss" attained in the previous section. In LHeC case, the spin direction will be kept vertical in the arcs, and therefore this scheme can be used without the alternating the chirp sign.
} 
collision in one of eRHIC's detectors, the electron beam is decelerated to $10 \mathrm{MeV}$ and is dumped. The path-length of the $30 \mathrm{GeV}$ beam-line provides for 180 degrees phase shift, and the used electrons decelerate at the same linacs in the reverse sequence. After reaching $0.6 \mathrm{GeV}$, they are bought to the injection ERL to recover the energy.

eRHIC's low emittance ERL lattice has natural chromaticity of $C_{x_{-} s}=-28.571 ; C_{y_{-} s}=-20.242$ per sextant. While mergers and combiners, as well as straight sections, add to natural chromaticity, their contribution is relatively small, i.e. can be just few units. It means that the total $360^{\circ}$ turn chromaticities can be estimated as $C_{x_{-} t} \cong-175 ; C_{y_{-} t} \cong-125$ and $\phi_{x_{-} t} \approx 1100 ; \phi_{y_{-} t} \approx 800^{10}$. Further more, total accumulated chromaticity for six passes up and five passes down in energy is $C_{x_{-} E R L} \sim-2000 ; C_{y_{-} E R L} \sim-1400$ and $\phi_{x_{-} E R L} \approx 1.25 \cdot 10^{4} ; \phi_{y_{-} E R L} \approx 8.5 \cdot 10^{3}$.

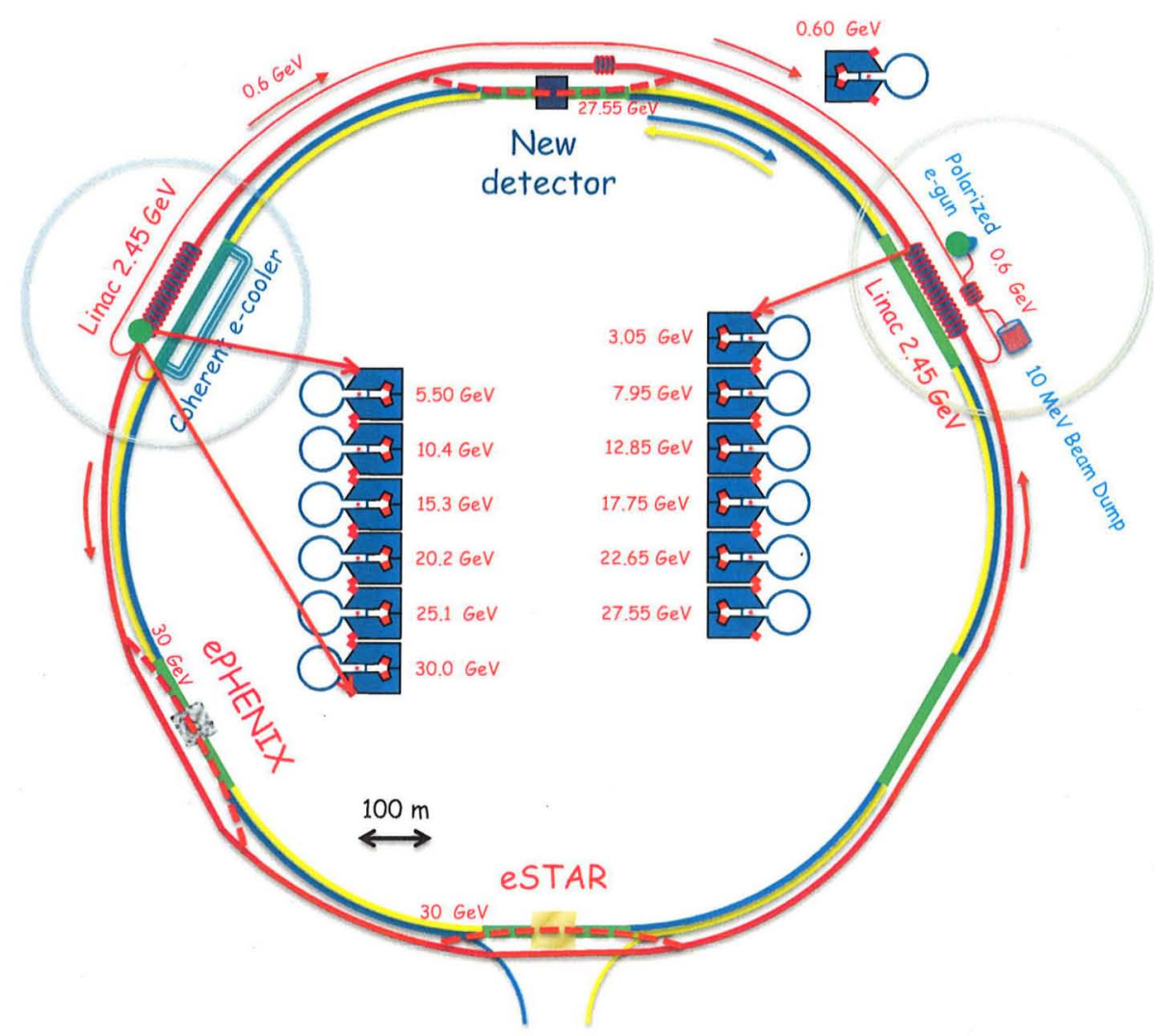

Figure 7. A sketch of eRHIC with $30 \mathrm{GeV}$ ERL. More about eRHIC design can be found in $[32,33]$

\footnotetext{
${ }^{10}$ The chromaticity is properly defined for a beam with constant energy $E$ and $\delta=\delta E / E$ being constant. In linacs, where energy changes and $\delta E$ is no longer a constant, traditional chromaticity should be redefined.
} 
Fig. 8 shows a BNL's version of $60-\mathrm{GeV}$ ERL for $\mathrm{LHeC}$ electron-hadron collider. It is comprised of a single pass $0.3 \mathrm{GeV}$ energy-recovery injector and two main $9.95 \mathrm{GeV}$ super-conducting RF linacs. Electrons pass three times through the main linacs on their way up to $60 \mathrm{GeV}$, and, after colliding with LHC protons, three times on the way down in the energy.

Low emittance ERL lattice for this ERL has natural chromaticity of $C_{x_{-} s}=-254.29 ; C_{y_{-} s}=-227.37$ per $180^{\circ}$ arc. Similarly to eRHIC ERL lattice, the mergers, combiners and straight sections add to natural chromaticity just few units. The chromaticities of the $360^{\circ}$ turn can be estimated as $C_{x_{-} t} \cong-510 ; C_{y_{-} t} \cong-460$ and $\phi_{x_{-}} \approx 3.2 \cdot 10^{3} ; \phi_{y_{-} t} \approx 2.9 \cdot 10^{3}$. Similarly, the total accumulated chromaticity on three passes up and two passes down is $C_{x_{-} E R L} \sim-2500 ; C_{y_{-} E R L} \sim-2300$ and $\phi_{x_{-} E R L} \approx 1.5 \cdot 10^{4} ; \phi_{y_{-} E R L} \approx 1.4 \cdot 10^{4}$.

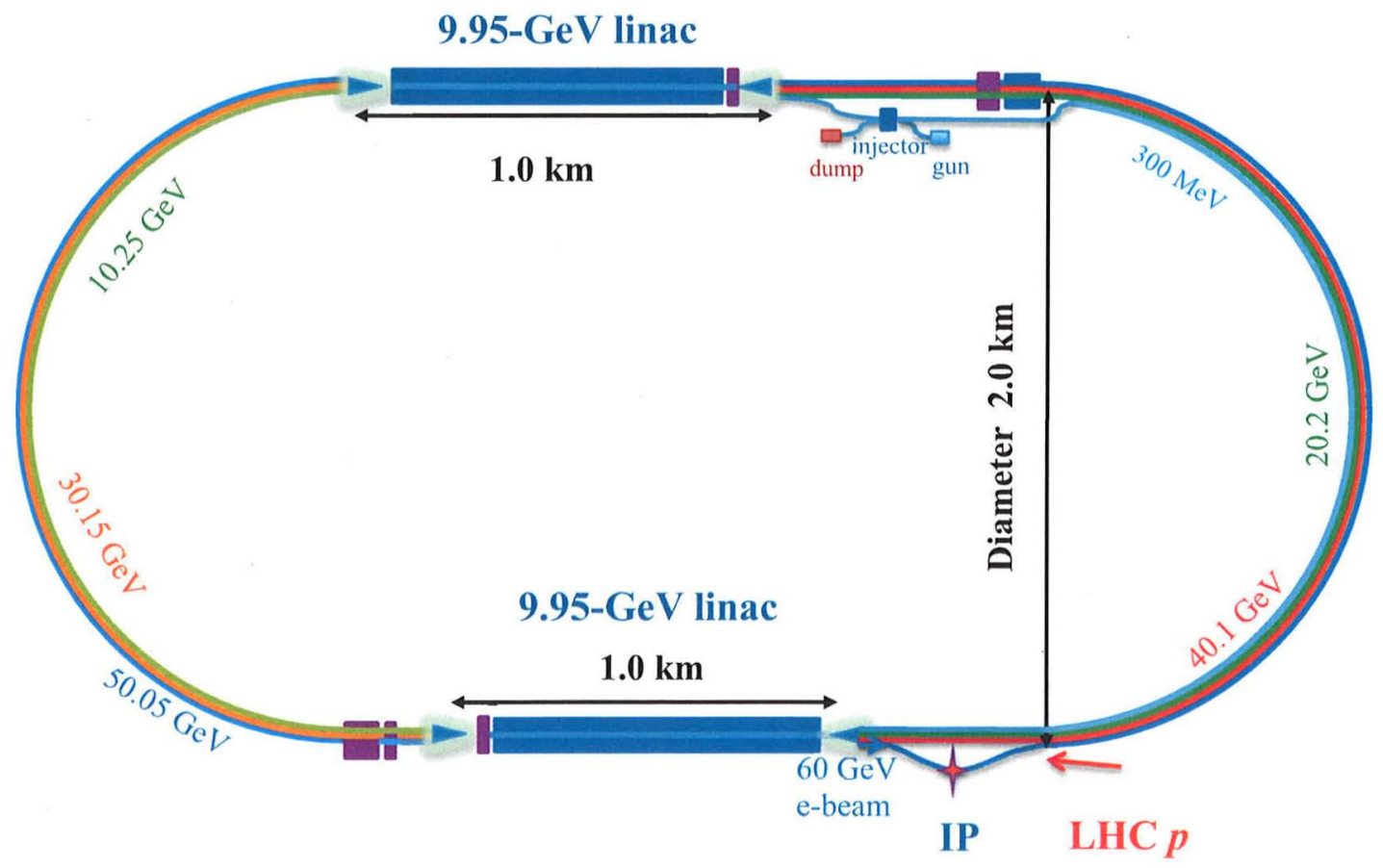

Figure 8. BNL's design of $60 \mathrm{GeV}$ ERL for LHeC linac-ring collider [35]

In contrast with the cumulative beam break-up, the TBBU ERL instability relies on the feed-back provided by electron bunches returning to the same RF cavity. In other words such feed-back occurs only after e-beam goes through a complete 360-degree turn. Hence, one turn chromaticity will play a dominant role in suppressing a single-turn TBBU. 
For a rough estimate of the TBBU suppression in the above ERLs, let's consider an electron beam with a Gaussian energy spread of $\sigma_{\delta}=5 \cdot 10^{-3} 11$. For eRHIC ERL, the vertical bunch response (i.e. a one turn response $\left\langle T_{12}(s|s+C\rangle)\right.$ will be suppressed by a factor of $2.9 \cdot 10^{3}$, while the horizontal suppression factor will be an astronomic factor of $3.7 \cdot 10^{6}$. Since chromaticity per turn is higher in the LHeC case, the suppression will be astronomical factor of $\sim 10^{45}$, i.e. other higher order effects and imperfection of Gaussian profile should be considered. It also means that in the LHeC case, $\sigma_{\delta} \cong 1.3 \cdot 10^{-3}$ will be sufficient for suppressing single-turn response by a factor of 1,000 .

Very large values of accumulated chromaticity $\phi \propto-10^{4}$ for a beam passing through the entire eRHIC or LHeC ERL means that an average $\sigma_{\delta} \sim 5 \cdot 10^{-4}$ will be sufficient to erase memory of low beam returning to the ERL injector, i.e. to suppress $\left\langle T_{12}\right.$ (in $|$ out $\rangle$ of the main ERL by three-to-four four orders of magnitude.

As discussed in Appendix $\mathrm{C}$, there are higher order effects, which can, in principle, reduce, or, at least, significantly modify prediction of eq. (17). To address this concern, I tested the concept by directly calculating a response of the beam with a Gaussian energy distribution propagating through a beam-line comprising of 1024 FODO cells. Each FODO cell is comprised of one focusing and one defocusing quadrupoles.

Hence I can use paraxial approximation for the particle's trajectory, it is very easy to write the exact analytical expression for the transport matrix of the FODO cell for an ultra-relativistic particle with arbitrary momentum $p=p_{o}(1+\delta): T_{F O D O}(\delta)$. The matrix is multiplied necessary number of times ${ }^{12}$

$$
T_{\text {total }}(\delta)=\left[T_{\text {FODO }}(\delta)\right]^{1024}
$$

to form exact matrix of the beam-line. The beam displacement at the end of the beam-line to an angular kick $\theta$ at its entrance will be a simple convolution:

$$
\langle x\rangle=\left\langle T_{12 \text { total }}\right\rangle \cdot \theta ; \quad\left\langle T_{12 \text { total }}\right\rangle=\int_{-\infty}^{\infty} T_{12 \text { total }}(\delta) f(\delta) d \delta,
$$

where $T_{12 \text { total }}(\delta)$ is an exact, analytical and nonlinear function of $\delta$. For comparison reason, I define the suppression factor of the transverse response as:

\footnotetext{
${ }^{11}$ As it is discussed above, such energy spread can be correlated and can introduced intentionally by off-cress acceleration of the electron bunch with Gaussian longitudinal profile. Furthermore, if needed, the sign of the energy chirp can alternate from pass to pass. The later does not affect the conclusions.

${ }^{12}$ Cell number $N=1024=2^{10}$ was chosen to reduce simulation time by reducing $N$ matrix multiplications to $\log _{2}(N)=10$ via $T^{2^{n+1}}=\left[T^{2^{n}}\right]^{2}$.
} 


$$
S=\frac{\max \left(\left|T_{12 \text { toral }}(\delta)\right|\right)}{\left|\int_{-\infty}^{\infty} T_{12 \text { total }}(\delta) f(\delta) d \delta\right|},
$$

Here I present results for a FODO lattice with equal focusing in both directions ${ }^{13}$. The exact transverse matrix of such FODO cell comprised of two quarupoles with length $l$, and the nominal strength $K_{1}$ and separated by two drift spaces with length $L$ is:

$$
\begin{gathered}
T_{\text {FODO }}(\delta)=\left[\begin{array}{ll}
1 & L \\
0 & 1
\end{array}\right] \cdot\left[\begin{array}{cc}
\cos \varphi & \kappa^{-1} \sin \varphi \\
-\kappa \sin \varphi & \cos \varphi
\end{array}\right] \cdot\left[\begin{array}{cc}
1 & L \\
0 & 1
\end{array}\right] \cdot\left[\begin{array}{cc}
\cosh \varphi & \kappa^{-1} \sinh \varphi \\
\kappa \sinh \varphi & \cosh \varphi
\end{array}\right] ; \\
\kappa(\delta)=\sqrt{\frac{K_{1}}{1+\delta}} ; \varphi(\delta)=\kappa(\delta) l .
\end{gathered}
$$

This exact matrix function was used for the direct numerical calculations of $T_{12 \text { total }}(\delta)$ of the beam-line and convolving it with Gaussian energy distribution (14) with relative RMS energy spread of $\sigma_{i}=2 \cdot 10^{-3}$. I did not observed any significant deviations for from expect theoretical behavior predicted by eq. (17) neither at a small values (as expected), not at large values of the of total beam-line chromaticity.

For example, the beam-line with tune advance of 0.22285 per cell has chromaticity of $C=-276.68$ and the calculated suppression factor 428 -fold, which compares favorably with theoretical estimation of 422 -fold given by eq. (17).

Increasing the chromaticity further to $C=-328.51$ by increasing tune advance per cell to 0.2499 , increased the calculated suppression factor to $5.9310^{3}$. It also reasonably close to the theoretical estimation of $5.0210^{3}$ given by eq. (17).

It is quite remarkable that at large values of the suppression, the exact value of the beam response suppression exceeds the theoretical predictions. Possible explanation of this phenomena is that high order effects increase particle's de-coherence, compared with first order chromatic effects. As can be seen from Fig. 9, the behavior of theof the chromatic suppression does not deviate strongly from first order chromatic behavior represented by eqs. (10) and (11).

The tune advance has mostly linear dependence on $\delta$ with quadratic term contributing $\Delta Q_{x}^{s n d} \cong 0.049$ at $\delta= \pm 0.01$. As expected, neither the $\beta$-function, nor the enevelope of $T_{12}$, do not significantly change within the $\delta= \pm 0.01$ range. The $\beta$-function changes only for less than $1 \%$ at $\delta=\sigma_{\delta}$ and a smooth envelope of oscillating $T_{12}$ provides for a strong suppression of the overall response when convoluted with smooth energy distributions.

13 Tests with non-equal strength of the quadrupole did not reveal any difference in behavior from this example. 
(a)

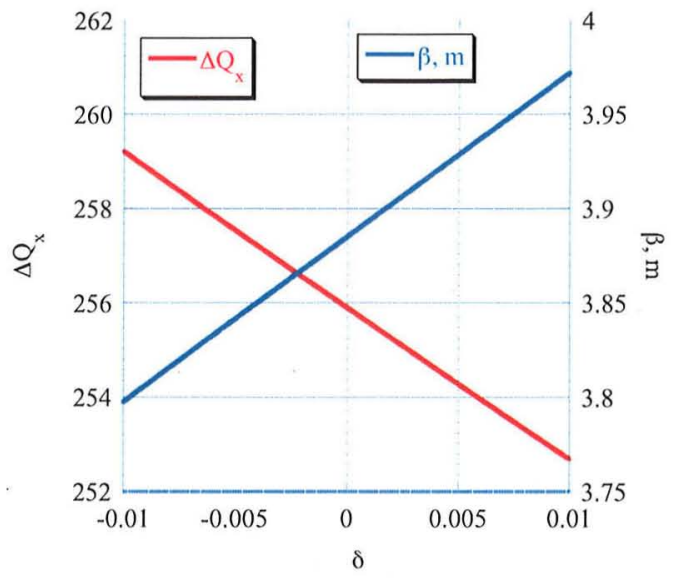

(b)

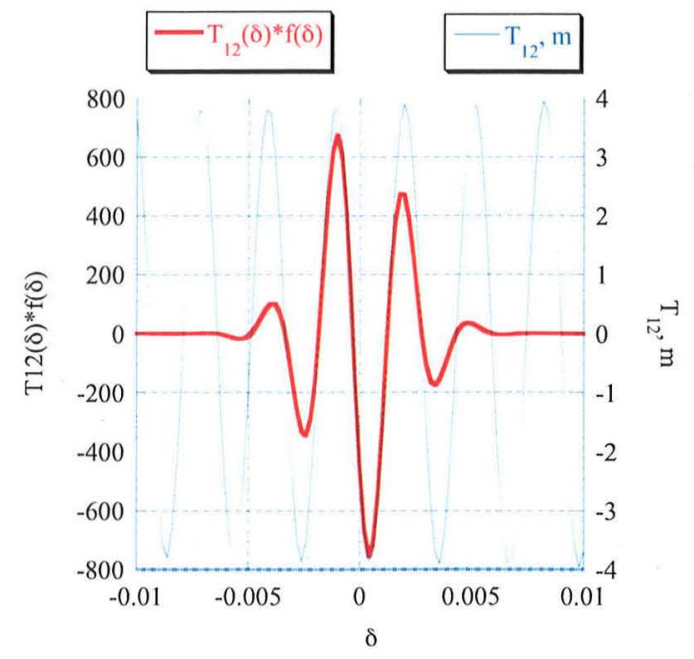

Figure 9. The FODO beam-line optics parameters as function of the particle momentum for a nominal tune advance per cell $\Delta Q_{x}(\delta=0)=0.2499$ : (a) Dependence of the total horizontal tune advance (red line) and beta-function (blue line) on the relative momentum deviation $\delta$; (b) Dependence of the beam-line's $\mathrm{T}_{12}$ element (blue line) and its convolution with the momentum distribution function (red line) on the relative momentum deviation $\delta$.

\section{An ultimate case.}

Let's consider an ERL comprising of a sequence of linacs and arcs (or transport channels): Linac1 $\rightarrow$ Arc1 $\rightarrow$ Linac2 $\rightarrow$ Arc2 ... When the product of the chromaticity and the energy spread in the arcs is very large $\left|\phi \sigma_{\delta}\right|>>1$, then the electron beam forgets the kick it received in the previous linac while traveling through an arc. In this case, the traditional ERL TBBU excitation scheme falls apart, and each linac sees fresh electron beams (to be exact, a combination two or more electron beams with various energies, both accelerating and decelerating). This means that the beam's stability problem is reduced to the traditional cumulative transverse instability in long linacs. The nature of this instability [31] differs from the ERL TBBU because there is no feed-back and the corresponding exponential growth. This well studied and higher-threshold (compared with TBBU) instability [32-34] is outside the scope of this paper.

In the TBBU theory [14-19], the low energy passes through the linac are considered to be most vulnerable to the TBBU instability. Specifically, the conclusion are that the TBBU threshold is proportional to the lowest energy in the recirculating path and it is also inverse proportional to number of the recirculating passes. Two examples described in the previous section, show that this rule is no longer applicable to an ERL where 
product of the chromaticity of betatron oscillations and the relative energy spread is significantly larger than unity.

Thus, in addition to improving stability of the beam, using highly chromatic lattices in ERLs allow to use cost-effective multi-pass ERLs as well as the very effective energy recovery by dumping the used electron beams at very low energies.

\section{Discussions and Conclusions}

I had shown in this paper that using the natural chromaticity of the arcs in ERLs affords an opportunity of suppressing the beam's transfer function (its response to transverse kicks), and either suppressing TBBU instability or significantly increasing allowable operating currents.

The proposed method paves the way for multi-pass ERLs becoming an economic choice for high-energy electron accelerators. Using the natural chromaticity of the ERL lattice offers a win-win case: It does not require sextupole magnets to compensate for chromaticity (i.e., reduces the cost of the accelerator) while increasing the threshold for TBBU instability. Still, in special cases of compact machines wherein natural chromaticity is small or using lattice with large natural chromaticity is impractical, one can consider installing sextupoles and intentionally increasing chromaticity to the desirable level. The fact that in ERLs electrons used only once, provide much-relaxed requirements on the linearity of the lattice, compared with storage rings, and use of very strong sextupoles could be possible.

In contrast to other mitigation methods, such as HOM damping, this method is straightforward, and can be established without significant investments into SRF technology. It also may allow us to use longer SRF linac strings, an increase in the realestate gradients in ERLs, and a lower injection energy. Overall, this method provides an additional tool in advancing ERL technology towards high-energy, high-current operations.

Standard TBBU simulation codes presently do not have capability of including chromatic effects in the simulation, with one possible exception [36]. While a verification of the proposed suppression mechanism by a direct TBBU simulations would be beneficial, such tests are going beyond scope of this paper.

The author would like to thank Ilan Ben Zvi, Yue Hao, Dmitry Kayran (BNL) and Eduard Pozdeyev (FRIB) for fruitful, in-depth discussions of the methods, and to Georg Hoffstaetter (Corbel University) and Frank Zimmermann (CERN) for their interest in the prospects of suppressing TBBU in high-energy ERLs.

The work was supported by Brookhaven Science Associates under Contract No. DEAC02-98CH10886 with the U.S. DOE 


\section{References}

[1] M. Tigner, A possible apparatus for electron clashing-beam experiments, Nuovo Cimento 37, 1228 (1965)

[2] G. R. Neil, C. L. Bohn, S. V. Benson, G. Biallas, D. Douglas, H. F. Dylla, R. Evans, J. Fugitt, A. Grippo, J. Gubeli, R. Hill, K. Jordan, R. Li, L. Merminga, P. Piot, J. Preble, M. Shinn, T. Siggins, R. Walker, and B. Yunn, Sustained Kilowatt Lasing in a Free-Electron Laser with Same-Cell Energy Recovery, Phys. Rev. Lett. 84 (2000) 662

[3] G. L. Carr, Michael C. Martin, Wayne R. McKinney, K. Jordan, George R. Neil, G. P. Williams, High-power terahertz radiation from relativistic electrons, Nature $\mathbf{4 2 0}$ (2002) 153

[4] S.M.Gruneret et al., Energy recovery linacs as synchrotron radiation sourcesReview of Scientific Instruments 73/3 (2002) 1402

[5] K.J. Kim, Y. Shvyd'ko, S. Reiche, A Proposal for an X-Ray Free-Electron Laser Oscillator with an Energy-Recovery Linac, Physical Review Letters 100 (2008) 244802

[6] I Ben-Zvi, Ya. Derbenev, V.N. Litvinenko and L. Merminga, Nucl. Instr. and Meth. Energy recovery linacs in high-energy and nuclear physics, A 557 (2006) 28

[7] V.N. Litvinenko, Y.S. Derbenev, Coherent Electron Cooling, Physical Review Letters $102(2009) 114801$

[8] V.N.Litvinenko, LHeC and eRHIC, In the Proc.of the 2009 Europhysics Conference on High Energy Physics, 16-22 July 2009 Krakow, Poland, http://www.bnl.gov/isd/documents/70608.pdf

[9] C M. Lyneis, M S. McAshan, R E. Rand, H. A. Schwettman, TI. Smith and J P. Turneaure, The Stanford Superconducting Recyclotron, IEEE Transactions on Nuclear Science, Vol. NS-26, No. 3, June 1979

[10] P.Axel, L.S.Cardman, H.D.Graef, A.O.Hanson, R.A.Hoffswell, D.Jamnik, D.C.Sutton, R.H.Taylor, and L.M.Young, Operating Experience with MUSL-2, IEEE Transactions on Nuclear Science, Vol. NS-26, No. 3, June 1979

[11] C.M. Lyneis, R.E. Rand, H.A. Schwettman, and A.M. Vetter, Standing wave model of regenerative beam breakup in recirculating electron accelerators, Nuclear Instruments and Methods 204 (1983) 269-28

[12] R.E. Rand, Recirculating electron accelerators (Harwood Academic Publishers, New York, 1984), section 9.5

[13] R.E. Rand and T.I. Smith, Beam optical control of beam breakup in a recirculating electron accelerator, Particle Accelerators, Vol. 11, pp. 1-13 (1980)

[14] G.A. Krafft, J.J. Bisognano, S. Laubach; Calculating Beam Breakup in Superconducting Linear Accelerators, Tech-Note CEBAF-PR-90-39, https://misportal.jlab.org/ul/publications/view pub.cfm?pub id $=1329$ 
[15] J.J. Bisognano, R.L. Gluckstern, in Proceedings of the 1987 Particle Accelerator Conference, Washington, DC (IEEE Catalog No. 87CH2387-9), pp. 1078-1080.

[16] G.A. Krafft, J.J. Bisognano, in Proceedings of the 1987 Particle Accelerator Conference, Washington, DC (IEEE Catalog No. 87CH2387-9), pp. 1356-1358.

[17] G.H. Hoffstaetter and I.V. Bazarov, Beam-breakup instability theory for energy recovery linacs, Phys. Rev. ST AB 7, 054401 (2004).

[18] E. Pozdeyev, Phys. Rev. ST Accel. Beams 8, 054401 (2005)

[19] G.H. Hoffstaetter, I.V. Bazarov and C. Song, Recirculating beam-breakup thresholds for polarized higher-order modes with optical coupling, Phys. Rev. ST Accel. Beams 10, (2007) 044401

[20] C.D. Tennant, K.B. Beard, D.R. Kouglas, K.C. Jordan, L.Merminga, E.G. Pozdeyev, T. Smith, First observations and suppression of multipass, multibunch beam breakup in the Jefferson Laboratory free electron laser upgrade, Phys. Rev. ST AB 8, 074403 (2005)

[21] H. Hahn, I. Ben-Zvi, R. Calaga, L. Hammons, V. N. Litvinenko, W. Xu, Physics Review Special Topics Accelerator and Beams 13, 121002 (2010)

[22] C.W. Leemann, D.R. Douglas, G.A. Krafft, Annu. Rev. Nucl. Part. Sci. 51 (2001) 413

[23] V.N. Litvinenko et al., High-energy high-luminosity electron-ion collider eRHIC, In "The EIC Science case: a report on the Joint BNL/INT/JLab program: Gluons and the quark sea at high energies: distributions, polarization, tomography, Editors: D. Boer, M. Diehl, R. Milner, R. Venugopal, W. Vogelsang, (2011), 438 http://arxiv.org/pdf/1108.1713v1

[24] F. Zimmermann et al., Proceedings of EPAC08, Genoa, Italy (2008) 2847

[25] R. Calaga et al., Nuclear Instruments and Methods in Physics Research A 557 (2006) 243

[26] W. Xu, I. Ben-Zvi, R. Calaga, H. Hahn, E.C. Johnson, J. Kewisch, Nuclear Instruments and Methods in Physics Research A 662 (2010) 17

[27] V. Shemelin et al., Proceedings of EPAC 2006, Edinburgh, Scotland (2006) 468

[28] H. Wiedeman, Particle Accelerator Physics II, Springer-Verlag, Berlin, 1995

[29] E.E.Courant, H.S.Snyder, "Theory of the alternating-gradient synchrotron", Annals of Physics, 3, 1, 1958

[30] D. Trbojevic et al., Proc. of Second International Particle Accelerator Conference, San Sebastian, Spain, September 4-9, 2011, p. 1099, http:/accelconf.web.cern.ch/AccelConf/IPAC2011/papers/tupc045.pdf

D. Trbojevic et al., 2011 Particle Accelerator Conference, New York, NY, USA, $\begin{array}{lllll}\text { March 25-April 2011, 1, 696, } & \text { p. }\end{array}$ http:/accelconf.web.cern.ch/AccelConf/PAC2011/papers/tuoan3.pdf

[31] R. B. Neal and W. H. Panofsky, Science 152, 1353 (1966) 
[32] R.H. Helm and G.A. Loew, Linear Accelerators (North-Holland, Amsterdam, 1970)

[33] W.K.H. Panofsky and M. Bander, Rev. Sci. Instrum. 39, 206 (1968)

[34] K.A. Thompson, R.D. Ruth, Physical Review D, 41 (1990) 964

[35] V.N. Litvinenko, Y. Hao, D. Kayran, V.Ptitsyn, D. Trbojevic, N. Tsoupas, ERL option for LHeC, January 2011, to be published

[36] G. Hoffstaetter, private communication, Cornell University ERL team discusses possibility to run such test using their code.

[37] V.N.Litvinenko, Selected Notes from the USPAS Winter 2008 Accelerator Physics Course, http://uspas.fnal.gov/materials/08UCSC/Accelerator Physics1.pdf

[38] V.N. Litvinenko, Analytical Tools in Accelerator Physics, C-A/AP/406 note, September 2010, BNL, http://www.cadops.bnl.gov/AP/ap notes/ap note 406.pdf

[39] A.A.Kolomensky, A.N.Lebedev, Theory of Cyclic Particle Accelerators, Wiley, New York, 1962

[40] N.Bogolyubov, Yu.A.Mitropolsky, "Asymptotic Methods in the theory of non-linear oscillations", Fizmatgiz, Moscow, 1977 


\section{Appendix A: Envelope and phase advance dependence on the particle energy}

Here I provide a very short $1-\mathrm{D}$ derivation of the envelope and phase variation for of-momentum particle. More details can be found in $[37,38]$.

Let's consider a Hamiltonian of transverse 1D linear motion of a particle with constant longitudinal momentum $p=p_{o}(1+\delta)$ in a beam-line with focusing function $K(s):^{14}$

$$
\mathbf{H}=\frac{p_{x}^{2}}{2}+\frac{K(s)}{1+\delta} \frac{x^{2}}{2}=\mathbf{H}_{o}-v K(s) \frac{x^{2}}{2},
$$

where $v=\delta /(1+\delta)$, which we assume to be a infinitesimally small $|v| \cong|\delta| \ll<$, and $p_{o}$ is the momentum of the reference particle. Let's us use know parametric solution for the reference particle [29],

$$
x=a \cdot \mathbf{w}_{o}(s) \cdot \cos \left(\psi_{o}(s)+\varphi\right) ; \beta_{o} \equiv \mathbf{w}_{o}^{2} ; \psi_{o}^{\prime}=1 / \beta_{o},
$$

where $a=\sqrt{2 I}, \varphi$ are determined by initial conditions. It is well known [39] that parameterization (A2) is a Canonical transformation from $\left\{x, x^{\prime}\right\}$ to the action-angle variables $\{\varphi, I\}$ with reduced Hamiltonian:

$$
h=\mathbf{H}-\mathbf{H}_{o}=-v I \cdot K(s) \mathbf{w}_{o}^{2}(s) \cdot \cos ^{2}\left(\psi_{o}(s)+\varphi\right),
$$

and reduced equations of motion:

$$
\begin{aligned}
& \varphi=\frac{\partial h}{\partial I}=-v \cdot K(s) \mathbf{w}_{o}^{2}(s) \cdot \cos ^{2}\left(\psi_{o}(s)+\varphi\right) ; \\
& I^{\prime}=-\frac{\partial h}{\partial \varphi}=-v \cdot I \cdot K(s) \mathbf{w}_{o}^{2}(s) \cdot \sin \left(2\left(\psi_{o}(s)+\varphi\right)\right),
\end{aligned}
$$

Using standard perturbation method [40] one can easily integrate this equations:

$$
\begin{aligned}
& \varphi(s)=\varphi_{o}-\delta \cdot \int_{0}^{s} K(z) \mathbf{w}_{o}^{2}(z) \cdot \frac{1+\cos \left(2\left(\psi_{o}(z)+\varphi_{o}\right)\right)}{2} d z+O\left(\delta^{2}\right) \\
& I=I_{o}\left\{1-\delta \cdot \int_{o}^{s} K(z) \mathbf{w}_{o}^{2}(z) \cdot \sin \left(2\left(\psi_{o}(z)+\varphi_{o}\right)\right) d z\right\}+O\left(\delta^{2}\right)
\end{aligned}
$$

From this result one already can see that in a periodic lattice (i.e. $\left.K(s)=K(s+C) ; \mathrm{w}_{0}(s+C)=\mathrm{w}_{0}(s)\right)$ the envelope (amplitude) variation has only an oscillating term (traditionally called a beta-beating), while the phase has both

${ }^{14}$ For example, $K_{y}(s)=\frac{e}{p_{o} c} \frac{\partial B_{y}}{\partial x}$ or $K_{x}(s)=-\frac{e}{p_{o} c} \frac{\partial B_{y}}{\partial x}+\left(\frac{e B_{y}}{p_{o} c}\right)^{2}$ 
monotonically growing and oscillating terms. Substituting (A5) into (A2) and keeping only first order terms of $v$ one gets:

$$
\begin{gathered}
x \cong a_{0} \cdot \mathbf{w}_{\varepsilon}(s) \cdot \cos \left(\psi_{o}(s)+\varphi(s)\right) \\
\mathbf{w}_{\varepsilon}(s)=\mathbf{w}_{o}(s)\left\{1-\frac{\delta}{2} \cdot \int_{0}^{s} K(z) \mathbf{w}_{o}^{2}(z) \cdot \sin \left(2\left(\psi_{o}(z)+\varphi_{o}\right)\right) d z\right\}+O\left(\delta^{2}\right) .
\end{gathered}
$$

Choice of the initial amplitude and phase, gives the required expressions in eqs. (10) and (11).

Finally, it is also well know that for a linear system the beam emittance is an average of the actions of individual particles:

$$
\varepsilon=\langle I\rangle
$$

and averaging second equation in (A5) over initial betatron phase $\varphi_{0}$ gives expected fact that emittance of the beam ${ }^{15}$ does not suffer from the chromaticity of the beam-line:

$$
\langle I\rangle=\left\langle I_{0}\right\rangle+O\left(\delta^{2}\right)
$$

The later is a well know experimental fact that chromaticity of the betatron oscillations does not result in emittance growth in storage ring, where particles propagate for millions of turns and accumulate astronomic value of the phase spread caused by chromaticity.

\section{Appendix B. Finite bunch length effect and HOM frequency}

Traditional TBBU treatment assume that electron bunch is much shorter than period of HOM oscillations. Frequently such analysis is sufficient.

Since I am considering a possibility of correlated energy spread, i.e. time dependent energy chirp, it is worth considering the details of the interaction of such a beam with a dipole HOM hawing frequency $\omega_{0}$. Let's consider a bunch of electron with RMS duration $\sigma_{r}$ passing through the cavity at the HOM phase of $\varphi_{o}$. A central electron will receive a transverse kick $x_{o}^{\prime}=\theta \cdot \sin \left(\varphi_{o}\right)$. After an interval $t$ such electron arrives to another cavity with displacement $x=T_{12} \cdot \theta \cdot \sin \left(\varphi_{0}\right)$ and excites EM field in the HOM with frequency $\omega_{1}$ proportional to $A \propto e R_{H O M} \cdot T_{12} \cdot \theta \cdot \sin \left(\varphi_{o}\right) \cdot \sin \left(\omega_{1} t\right)$. If electron bunch is very short compared with the HOM oscillation period, $\omega_{o} \sigma_{t} \ll 1 ; \omega_{1} \sigma_{t} \ll 1$, all $N$ electrons will excite the EM wave coherently and the total excited amplitude would be $A \propto q R_{H O M} \cdot\left\langle T_{12}\right\rangle \cdot \theta \cdot \sin \left(\varphi_{o}\right) \cdot \sin \left(\omega_{1} t\right) ; q=N e$. Otherwise one should consider effect of the finite bunch length on the bunch response.

\footnotetext{
${ }^{15}$ In this case we naturally assume that particles in the initial beam are evenly distributed over its betatron phases, i.e. the phase space density is a function only of the amplitude.
} 
An electron deviated from the bunch center by $\tau$ to would have kick $x_{o}^{\prime}=\theta \cdot \sin \left(\varphi_{o}+\omega_{o} \tau\right)$ and would excite the second cavity HOM as follows:

$$
A \propto e R_{H O M} \cdot T_{12}(\delta) \cdot \theta \cdot \sin \left(\varphi_{o}+\omega_{0} \tau\right) \cdot \sin \left(\omega_{1}(t+\tau)\right) .
$$

It is worth rewriting it in a following form:

$$
A \propto T_{12}(\delta) \cdot\left\{\cos \left(\varphi_{0}-\omega_{1} t+\left(\omega_{0}-\omega_{1}\right) \tau\right)-\cos \left(\varphi_{0}+\omega_{1} t+\left(\omega_{0}+\omega_{1}\right) \tau\right)\right\} .
$$

In the absence of the correlation between the energy deviation $\delta$ and $\tau$, and a Gaussian bunch length distribution $f(\tau)=\exp \left(-\left(\tau / \sigma_{t}\right)^{2} / 2\right) / \sqrt{2 \pi \sigma_{t}}$, averaging of (B.2):

$$
\langle A\rangle \propto\left\langle T_{12}\right\rangle \cdot\left\{e^{-\frac{\left(\omega_{0}-\omega_{1}\right)^{2} \sigma_{1}^{2}}{2}} \cos \left(\varphi_{0}-\omega_{1} t\right)-e^{-\frac{\left(\omega_{0}+\omega_{1}\right)^{2} \sigma_{1}^{2}}{2}} \cos \left(\varphi_{0}+\omega_{1} t\right)\right\} .
$$

While second term can vanish when $\sigma_{1}>1 /\left(\omega_{1}+\omega_{0}\right)$, it is not necessarily correct for the first term, especially if $\omega_{1} \cong \omega_{o}$ :

$$
\langle A\rangle \propto\left\langle T_{12}\right\rangle \cdot\left\{\cos \left(\varphi_{0}-\omega_{0} t\right)-e^{-2 \omega_{0}^{2} \sigma_{t}^{2}} \cos \left(\varphi_{0}+\omega_{o} t\right)\right\} .
$$

In the case of correlated energy spread with $\delta=\mu \tau ; \sigma_{\delta}=\mu \sigma_{1}$, one should use $T_{12}(\delta)=\mathrm{w}_{\mathrm{i} 0} \mathrm{w}(s, \delta) \sin \psi(s, \delta)$ and eq. (10). Integration is trivial and will result appearance of four exponential terms:

$$
\exp \left[\frac{\left(\phi \pm \zeta_{1,2}\right)^{2} \sigma_{\delta}^{2}}{2}\right] ; \zeta_{1,2}=\frac{\omega_{0} \pm \omega_{1}}{\mu}
$$

i.e. the exponential chromatic suppression will be valid when $|\phi|>>\left|\zeta_{1,2}\right|$. In other terms, for most interesting case of $\left|\phi \sigma_{\delta}\right|>>1$ the suppression projected by eq. (16) is correct for any HOM whose period is comparable or longer than the bunch length.

\section{Appendix C: Alternative perturbation method}

This is a simplified version of an arbitrary case, which can be found in $[37,38]$. For an $1 \mathrm{D}$ linear system with Hamiltonian $\mathbf{H}=X^{T} \cdot H \cdot X ; X^{T}=\left\{x, p_{x}\right\}$, there is a full set of two eigen vectors satisfying following equations and symplectic relations:

$$
\begin{gathered}
\tilde{Y}^{\prime}=D(s) \tilde{Y} \quad ; D=\sigma \cdot H ; \sigma=\left[\begin{array}{cc}
0 & 1 \\
-1 & 0
\end{array}\right] ; \\
\tilde{Y}^{*}=D(s) \tilde{Y}^{*} \\
\tilde{Y}=\tilde{Y} e^{i \psi}=\left[\begin{array}{c}
\mathrm{W} \\
(\mathrm{w}+i / \mathrm{w}) e
\end{array}\right] e^{i \psi ;} ; \quad \tilde{Y}^{* T} \sigma \tilde{Y}=Y^{* T} \sigma Y=2 i ;
\end{gathered}
$$


with obvious $A^{T} \sigma A \equiv 0, \forall A$. Let's consider a slightly perturbed Hamiltonian with respect to that for an ideal case (in this case a particle with designed energy) $H=H_{o}+\varepsilon h ; D=D_{o}+\varepsilon \Delta ;|\varepsilon| \ll<$. With the known eigen vectors for the ideal case:

$$
\begin{aligned}
& \tilde{Y}_{o}=\left[\begin{array}{c}
\mathrm{w}_{0} \\
\left(\mathrm{w}_{0}+i / \mathrm{w}_{0}\right)
\end{array}\right] e^{i \psi \psi_{0}} ; \tilde{Y}_{o}^{*}=\left[\begin{array}{c}
\mathrm{w}_{0} e^{-i \psi_{0}} \\
\left(\mathrm{w}_{0}-i / \mathrm{w}_{0}\right) e^{-i \psi_{0}}
\end{array}\right] ; \\
& \tilde{Y}_{0}^{\prime}=D_{0}(s) \tilde{Y}_{0} ; \quad \tilde{Y}_{o}^{*}=D_{0}(s) \tilde{Y}_{0}^{*} ; \quad D_{0}(s)=\left[\begin{array}{cc}
0 & 1 \\
-K(s) & 0
\end{array}\right] ;
\end{aligned}
$$

one can use perturbation theory similar to that used in quantum mechanics (see p.63 of [37]) to find perturbed solutions:

$$
\begin{gathered}
D(s)=D_{o}(s)+\varepsilon \cdot \Delta(s) \\
\tilde{Y}=a \tilde{Y}_{o}+b \tilde{Y}_{o}^{*} ;|b|=O(\varepsilon) \ll<|a|
\end{gathered}
$$

Using symplectic normalization of $\tilde{Y}^{*} S \tilde{Y}=\left(a^{*} \tilde{Y}_{o}^{T^{*}}+b^{*} \tilde{Y}_{o}^{T}\right) S\left(a \tilde{Y}_{o}+b \tilde{Y}_{o}^{*}\right)=2 i\left(|a|^{2}+|b|^{2}\right)$ imposed by $(\mathrm{C} 1)$, one finds that $|a|=1-O\left(\varepsilon^{2}\right)$.

It is important that to note that even though $|a|$ does not significantly deviates from unity, the complex amplitude $a=|a| e^{i x}$ can significantly deviate from $a=1$.

Keeping only first order terms in (C3) one finds a linear equation for $a$ and $b$ :

$$
\begin{gathered}
\tilde{Y}^{\prime}=\left\{D_{o}(s)+\varepsilon \Delta(s)\right\} \tilde{Y} ; \tilde{Y}_{o}^{\prime}=D_{o}(s) \tilde{Y}_{o} ; \\
a^{\prime} \tilde{Y}_{o}+b^{\prime} \tilde{Y}_{o}^{*}=\varepsilon \Delta(s)\left(a^{\prime} \tilde{Y}_{o}+b^{\prime} \tilde{Y}_{o}^{*}\right) ; \\
a^{\prime} \tilde{Y}_{o}+b^{\prime} \tilde{Y}_{o}^{*}=\varepsilon \Delta(s) a \tilde{Y}_{o}+O\left(\varepsilon^{2}\right) .
\end{gathered}
$$

Multiplying last equation from the left by the mode projection operators $\tilde{Y}_{0}^{T^{*}} \sigma$ : ,$\tilde{Y}_{o}^{T} \sigma$ : and using obvious $\sigma^{2}=-\mathbf{1}$, one gets linear equations

$$
\begin{gathered}
\tilde{Y}_{o}^{T^{*}} \sigma: \rightarrow 2 i a^{\prime} \cong-a \cdot \varepsilon\left(\tilde{Y}_{o}^{T^{*}} h(s) \tilde{Y}_{o}\right) \\
\tilde{Y}_{o}^{T} \sigma: \rightarrow-2 i b^{\prime} \cong-a \cdot \varepsilon\left(\tilde{Y}_{o}^{T} h(s) \tilde{Y}_{o}\right),
\end{gathered}
$$

which easily can be solve analytically.

In our specific case of equation of motions for a particle with a small energy deviation

$$
\varepsilon=\delta ; h(s)=\left[\begin{array}{cc}
-K(s) & 0 \\
0 & 0
\end{array}\right]
$$

the equations 


$$
\frac{a^{\prime}}{a}=-i \delta \cdot K(s) \mathrm{w}_{0}^{2}(s) ; \quad b^{\prime}=i a \frac{\delta}{2} \cdot K(s) \mathrm{w}_{0}^{2}(z) e^{2 i \psi_{0}(s)}
$$

can be easily solved:

$$
\begin{aligned}
& a(s)=e^{i \chi(s)} ; \chi(s)=-\frac{\delta}{2} \int_{0}^{s} K(z) \mathrm{w}_{0}^{2}(z) d z ; \\
& b=\frac{i \delta}{2} \int_{0}^{s} e^{i\left(2 \psi_{s}(z)+\chi(z)\right)} K(z) \mathrm{w}_{0}^{2}(z) d z ;
\end{aligned}
$$

where I use natural initial conditions of $a(0)=1 ; b(0)=0$. From this result one already can see that in a periodical lattice structure (i.e. $K(s)=K(s+C) ; Y(s+C)=Y(s)$ ) the error in phase of the betatron oscillations $\chi$ grows monotonically with the azimuth, while the deviation from the ideal eigen vector oscillates with double betatron frequency.

Combining this with (C2) we can write explicit expressions for linear part of modification to the envelope and the phase of the betatron motion:

$$
\mathrm{w}(\mathrm{s}) \cong \mathrm{w}_{0}(\mathrm{~s})(1+\delta \cdot v(s)) ; \psi_{o}(s)=\psi_{o}(s)+\delta \cdot \phi(s) ;
$$

using straight-forward manipulations:

$$
\begin{aligned}
& \mathrm{W}=\sqrt{\mathrm{W}_{\mathrm{o}}^{2}\left|a e^{i \psi \psi_{o}(s)}+b e^{-i \psi_{o}(s)}\right|^{2}} \cong \mathrm{W}_{\mathrm{o}}^{2}\left(1+\operatorname{Re} b(s) e^{-i\left(2 \psi_{o}(s)+\chi(s)\right)}\right) \\
& \psi(s)=\operatorname{Arg}\left(a e^{i \psi_{o}(s)}+b e^{-i \psi_{o}(s)}\right) \cong \psi_{o}(s)+\chi(s)+\operatorname{Im}\left(b e^{-i\left(2 \psi_{\psi_{o}}(s)+\chi(s)\right)}\right)
\end{aligned}
$$

One can easily see that the first order terms are identical to that derived in Appendix A.

For completeness, let me mention that in the case of an arbitrary linear betatron coupling, the system is fully described by the full set of four four-component eigen vectors [37,38], comprised of two complex conjugated pairs $\left\{Y_{1}, Y_{1}^{*}, Y_{2}, Y_{2}^{*}\right\}, \tilde{Y}_{k}=Y_{k}(s) e^{i \eta_{k}(s)} ; k=1,2$ :

$$
\begin{gathered}
\tilde{Y}_{1,2}^{\prime}=D(S) \tilde{Y} ; D=S \cdot H ; S=\left[\begin{array}{cc}
\sigma & 0 \\
0 & \sigma
\end{array}\right] \\
\tilde{Y}_{i}^{* T} S \tilde{Y}_{k}=Y^{* * T} S Y=2 i \delta_{i k} ; Y_{i}^{T} S Y_{k}=0
\end{gathered}
$$

where $\delta_{i k}$ is the Kronecker's delta. For a linear perturbation, I can expand any vector using full set of unperturbed eigen vectors:

$$
\begin{gathered}
D(s)=D_{o}(s)+\varepsilon \cdot \Delta(s) ; \\
\tilde{Y}_{k}=a_{k} \tilde{Y}_{o k}+b_{k} \tilde{Y}_{o k}^{*}+c_{k} \tilde{Y}_{o i}+d_{k} \tilde{Y}_{o i}^{*} ; i \neq k ;\left|b_{k}\right|,\left|c_{k}\right|,\left|d_{k}\right|=O(\varepsilon) \ll\left|a_{k}\right| \cong 1
\end{gathered}
$$

with solutions obtained using symplectic projection operators: 


$$
\begin{aligned}
\tilde{Y}_{k o}^{T *} S: & \rightarrow 2 i a_{k}^{\prime} \cong-a_{k} \cdot \varepsilon\left(\tilde{Y}_{k o}^{T^{*}} h(s) \tilde{Y}_{k o}\right) ; \\
\tilde{Y}_{k o}^{T} S: & \rightarrow-2 i b_{k}^{\prime} \cong a_{k} \cdot \varepsilon\left(\tilde{Y}_{k o}^{T} h(s) \tilde{Y}_{k o}\right) ; \\
\tilde{Y}_{i o}^{T^{*}} S & : \rightarrow 2 i c_{k}^{\prime} \cong a_{k} \cdot \varepsilon\left(\tilde{Y}_{i o}^{T *} h(s) \tilde{Y}_{k o}\right) ; \\
\tilde{Y}_{i o}^{T} S: & \rightarrow-2 i b_{k}^{\prime} \cong a_{k} \cdot \varepsilon\left(\tilde{Y}_{i o}^{T} h(s) \tilde{Y}_{k o}\right) ;
\end{aligned}
$$

which should be evaluated in exactly identical manner to that in (A7) for two pairs of $\{k, i\}:\{k=1, i=2\}$ and $\{k=2, i=1\}$. After straight forward calculations [37,38] one can find, that similar to decoupled case, the phase advance in a periodic lattice is a monotonic function of s:

$$
a_{k}=a_{k o} e^{i \chi_{k}(s)} ; \quad \chi_{k}(s)=-i \int_{0}^{s} Y_{k o}^{T^{*}}(z) h(z) Y_{k o}(z) d z ; k=1,2
$$

since in the periodic lattice the eigen vectors are periodic $Y_{k 0}(z+C)=Y_{k 0}(z)$ and the scalar $y=Y_{k}^{T^{*}} h Y_{k}$ under the integral is a real number:

$$
\begin{aligned}
& \text { 1. } y^{T} \equiv y \Rightarrow\left(Y_{k}^{T^{*}} h Y_{k}\right)^{T}=Y_{k}^{T^{*}} h Y_{k} ; \\
& \text { 2. } y^{*}=\left(Y_{k}^{T^{*}} h Y_{k}\right)^{*}=Y_{k}^{T} h Y_{k}^{*}=\left(Y_{k}^{T^{*}} h Y_{k}\right)^{T}=Y_{k}^{T^{*}} h Y_{k}=y .
\end{aligned}
$$

where use the fact that the Hamiltonian matrix is real $h^{*}=h$ and symmetric $h^{T}=h$.

In contrast to the traditional perturbation methods, used in Appendix A, the approach described in this section is applicable only for linear system. At the same time, it is not limited to the second order. Similarly to quantum mechanics, except for some pathological and degenerated cases (such as a parametric resonance of one cell), this perturbation series can be extended to an arbitrary order. 